\title{
Terminal Falling Characteristics of a Sand Grain with Dufour and Soret Effects
}

\author{
R. Nasrin ${ }^{*}$ and M. A. Alim \\ Department of Mathematics, Bangladesh University of Engineering \& Technology, Dhaka -1000, \\ Bangladesh
}

Received 1 March 2012, accepted in final revised form 16 September 2012

\begin{abstract}
The transient effect of double diffusive natural convection of flow in a differentially heated sand grain with Soret and Dufour coefficients is studied numerically. The right vertical wall has constant temperature $T_{c}$. The lower inlet opening is heated uniformly with fixed temperature $T_{h}$ and the velocity at the inlet of the fluid domain is set to the falling velocity $V_{i}$. The condition $T_{h}>T_{c}$ is maintained all over the domain. The concentration in right wall is maintained higher than inlet opening $\left(C_{c}<C_{h}\right)$. The governing equations are solved numerically subject to appropriate boundary conditions by a penalty finite-element method. Solutions are obtained for fixed Prandtl number $(\operatorname{Pr}=1.73)$, Rayleigh number $\left(R a=10^{4}\right)$, Dufour coefficient $\left(D_{f}=0.5\right)$ and Soret coefficient $\left(S_{r}=0.5\right)$. Transient analysis of the streamlines, isotherms, iso-concentration, falling velocity and forces on sand grain, the local and average Nusselt number and Sherwood number, temperature and concentration at subdomain centre as well as average form, subdomain horizontal and vertical velocities are presented graphically. It is found that the rate of heat transfer and mass transfer in the sand grain enhances and reduces respectively for shorter time periods and then they become almost steady.
\end{abstract}

Keywords: Sand grain; Natural convection; Transient; Dufour and Soret; Heat transfer.

(C) 2013 JSR Publications. ISSN: 2070-0237 (Print); 2070-0245 (Online). All rights reserved.

doi: http://dx.doi.org/10.3329/jsr.v5i1.10003 J. Sci. Res. 5 (1), 91-103 (2013)

\section{Introduction}

The mixed convection in enclosures continues to be a very active area of research during the past few decades. While a good number of works have made significant contributions for the development of the theory, an equally good number of works have been devoted to many engineering applications that include electronic or computer equipment, thermal energy storage systems etc.

\footnotetext{
*Corresponding author: rehena@math.buet.ac.bd
} 
Mixed convection heat transfer is an important phenomenon in engineering systems due to its wide applications in electronic cooling, heat exchangers, and thermal systems. Enhancement of heat transfer in such systems is very essential from the industrial and energy saving perspectives. For a detailed survey of literature on the natural convection heat transfer was given by Davis [1] and Ostrach [2]. However, we shall refer to a few important works that may serve as background for the present work. Transient flow field and heat transfer behavior of cold water in an enclosure is numerically investigated by Chang and Yang [3]. Ho and Tu [4] also studied transient flow of water. Osorio et al. [5] analyzed experimentally and numerically the natural convection of water near its density inversion in a square enclosure.

Double diffusive convection of water has been studied by Sezai and Mohamad [6] and Sivasankaran and Kandaswamy [7, 8]. In most of the problems, Soret and Dufour effects are assumed to be negligible. But the present work investigates both the effects. The diffusion of matter caused by temperature gradients (Soret effect) and diffusion of heat caused by concentration gradients (Dufour effect) become very significant when the temperature and concentration gradients are very large. Generally these effects are considered as second order phenomenon. The effects may become important in some applications such as the solidification of binary alloys, groundwater pollutant migration, chemical reactors, and geosciences.

Joly et al. [9], Bahloul et al. [10], Mansour et al. [11], Patha et al. [12] and Platten [13]. Joly et al. [9] showed the Soret effect on natural convection in a vertical enclosure. They investigated the particular situation where the buoyancy forces induced by the thermal and solutal effects are opposing each other and of equal intensity. Double diffusive and Soret induced convection in a shallow horizontal enclosure is analytically and numerically studied by Bahloul et al. [10] and also studied numerically by Mansour et al. [11]. They found that the Nusselt number decreased in general with the Soret parameter while the Sherwood number increased or decreased with this parameter depending on the temperature gradient induced by each solution.

It is essential to study heat transfer in an enclosure with partially heated active walls. Only a few studies are reported in the literature concerning heat transfer in enclosures with partially heated side walls, by Frederick and Quiroz [14], Erbay et al. [15] and Oztop [16]. Natural convection in an enclosure with partially active walls was studied by Nithyadevi et al. [17 - 19] and Kandaswamy et al. [20] without Soret and Dufour effects. Chamkha [21] analyzed heat and mass transfer for a non-Newtonian fluid flow along a surface embedded in a porous medium with uniform wall heat and mass fluxes and heat generation or absorption. The influence of the concentration to thermal buoyancy ratio, power-law fluid viscosity index, mixed convection parameter, suction or injection parameter, dimensionless heat generation or absorption parameter and the Lewis number on the local Nusselt and the Sherwood numbers was conducted in his study. Teamah [22] numerically performed double diffusive natural convection in rectangular enclosure in the presences of magnetic field and heat source. The author considered laminar regime under steady state 
condition and presented the predicted results for the average Nusselt and Sherwood numbers for various parametric conditions.

The present study deals with the transient natural convection of flow in a differentially heated sand grain in the presence of solute concentration with Soret and Dufour effects. The first stop for polluted water entering a water work is normally a large tank, where large particles are left to settle. More generally, gravity settling is an economical method of separating particles. If the fluid in the tank is moving at a controlled low velocity, the particles can be sorted in separate containers according to the time it takes for them reach the bottom. Therefore, transient free convection also becomes a crucial point of this study. The main issues discussed in this paper are: convective falling velocity, heat and mass transfer characteristics of a sand grain.

\section{Model Definition}

Fig. 1 shows a schematic diagram of a differentially heated sand grain. This model simulates a sand grain falling in water. The grain accelerates from standstill and rapidly reaches its terminal velocity. The model is an axially symmetric fluid-flow simulation in a moving coordinate system, coupled to a partial differential equation (PDE) for the force balance of the particle describing the grain's motion. Due to axial symmetry, the flow can be modeled in $2 \mathrm{D}$ instead of $3 \mathrm{D}$.
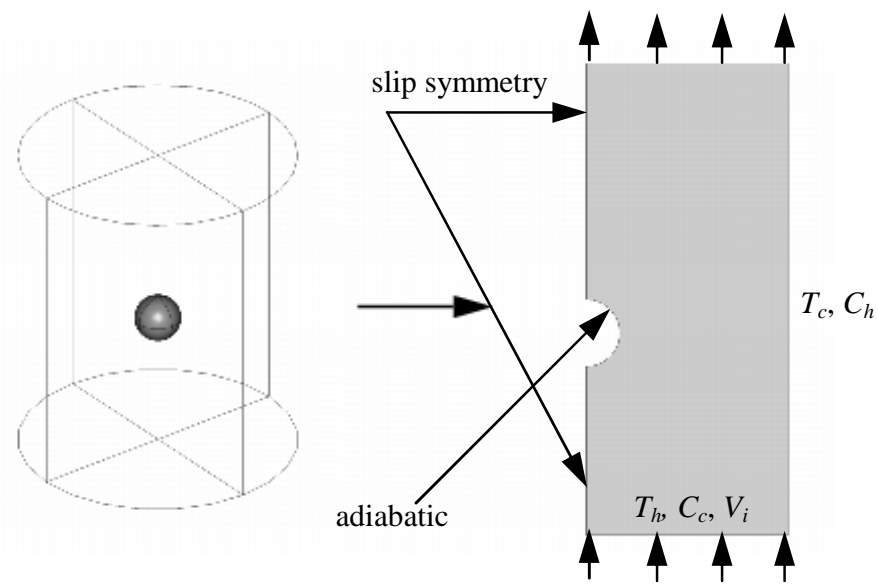

Fig. 1. Modeling domain of the sand grain.

The problem is solved in the accelerating reference system of the sand grain. This means that the volume force density $F$ in the Navier-Stokes equation is $F_{x}=0, F_{y}=-\rho a g$, where $a$ is the acceleration of the grain and $g$ is the acceleration due to gravity. 
The force balance is $m x=F_{g}+F_{y}$, where $m$ denotes the mass of the particle, $x$ the position of the particle, $F_{g}$ the gravitational force, and $F_{y}$ the $y$ component of the force that the water exerts on the sand grain.

The gravitational force is $F_{g}=-\rho_{\text {grain }} v_{\text {grain }} g$, where $v_{\text {grain }}$ is the volume of the sand grain and $\rho_{\text {grain }}$ its density.

The force that the water exerts on the grain is calculated by integrating the normal component of the stress tensor over the surface of the particle. Since the model is axially symmetric, the force will only have a $y$ component.

The dimensionless governing equations for transient natural convection in a sand grain in terms of the Navier-Stokes and energy equation are given as [23-25]:

$$
\begin{aligned}
& \frac{\partial U}{\partial X}+\frac{\partial V}{\partial Y}=0 \\
& \frac{\partial U}{\partial \tau}+U \frac{\partial U}{\partial X}+V \frac{\partial U}{\partial Y}=-\frac{\partial P}{\partial X}+\operatorname{Pr}\left(\frac{\partial^{2} U}{\partial X^{2}}+\frac{\partial^{2} U}{\partial Y^{2}}\right) \\
& \frac{\partial V}{\partial \tau}+U \frac{\partial V}{\partial X}+V \frac{\partial V}{\partial Y}=-\frac{\partial P}{\partial Y}+\operatorname{Pr}\left(\frac{\partial^{2} V}{\partial X^{2}}+\frac{\partial^{2} V}{\partial Y^{2}}\right)+\operatorname{Pr} R a_{T}(\theta-N r C) \\
& \frac{\partial \theta}{\partial \tau}+U \frac{\partial \theta}{\partial X}+V \frac{\partial \theta}{\partial Y}=\frac{1}{\operatorname{RePr}}\left(\frac{\partial^{2} \theta}{\partial X^{2}}+\frac{\partial^{2} \theta}{\partial Y^{2}}\right)+D_{f}\left(\frac{\partial^{2} C}{\partial X^{2}}+\frac{\partial^{2} C}{\partial Y^{2}}\right) \\
& \frac{\partial C}{\partial \tau}+U \frac{\partial C}{\partial X}+V \frac{\partial C}{\partial Y}=\frac{1}{R e S C}\left(\frac{\partial^{2} C}{\partial X^{2}}+\frac{\partial^{2} C}{\partial Y^{2}}\right)+S_{r}\left(\frac{\partial^{2} \theta}{\partial X^{2}}+\frac{\partial^{2} \theta}{\partial Y^{2}}\right)
\end{aligned}
$$

where $R e=\frac{V_{i} L}{v}, N r=\frac{R a_{c}}{R a_{T}}, \operatorname{Pr}=\frac{v}{\alpha}, R a_{T}=\frac{g \beta L^{3}\left(T-T_{c}\right)}{v^{2}}, \quad R a_{s}=\frac{g \beta L^{3}\left(c-C_{c}\right)}{v^{2}}, \quad S c=\left(\frac{v}{D}\right)$, $D_{f}=\frac{D k_{T}\left(C_{h}-C_{c}\right)}{C_{s} C_{p} v\left(T_{h}-T_{c}\right)}$ and $S_{r}=\frac{D k_{T}\left(T_{h}-T_{c}\right)}{T_{m} v\left(C_{h}-C_{c}\right)}$ are the Reynolds number, buoyancy ratio, Prandtl number, thermal and Solutal Rayleigh numbers, Schmidt number, Dufour and Soret coefficients respectively.

The corresponding boundary conditions take the following form:

At the inlet boundary: $V=1, \theta=1, C=0, U=0$,

at the right vertical boundary: $\theta=0, C=1, U=V=0$,

at the circular boundaries: $\frac{\partial \theta}{\partial N}=0, \frac{\partial C}{\partial N}=0, U=V=0$,

at the top surface convective boundary condition: $P=0$,

at left vertical boundary slip symmetry condition is applied.

The above equations are non-dimensionalized by using the following dimensionless dependent and independent variables:

$$
X=\frac{x}{L}, \quad Y=\frac{y}{L}, \quad U=\frac{u}{V_{i}}, \quad \tau=\frac{t}{V_{i}}, V=\frac{v}{V_{i}}, P=\frac{p}{\rho V_{i}^{2}}, \theta=\frac{T-T_{c}}{T_{h}-T_{c}}, C=\frac{c-C_{c}}{C_{h}-C_{c}}
$$


The local Nusselt and Sherwood numbers at the heated surface of the sand grain may be expressed, respectively as $N u_{\text {local }}=-\left.\frac{\partial \theta}{\partial Y}\right|_{Y=0}$ and $S h_{\text {local }}=-\left.\frac{\partial C}{\partial X}\right|_{X=1}$.

The average Nusselt and Sherwood numbers are $N u=\int_{0}^{1} N u_{\text {local }} d Y$ and $S h=\int_{0}^{1} S h_{\text {local }} d X$, respectively

\section{Numerical Implementation}

The Galerkin finite element method [26, 27] is used to solve the non-dimensional governing equations along with boundary conditions for the considered problem. The equation of continuity has been used as a constraint due to mass conservation and this restriction may be used to find the pressure distribution. The penalty finite element method [28] is used to solve the Eqs. (2) - (5), where the pressure $P$ is eliminated by a penalty constraint $\xi$, and the incompressibility criteria given by Eq. (1) which can be expressed as

$$
P=-\xi\left(\frac{\partial U}{\partial X}+\frac{\partial V}{\partial Y}\right)
$$

The continuity equation is automatically fulfilled for large values of $\xi$. Then the velocity components $(U, V)$, temperature $(\theta)$ and concentration $(C)$ are expanded using a basis set $\{\Phi\}_{k=1}^{N}$ as

$$
U \approx \sum_{k=1}^{N} U_{k} \Phi_{k}(X, Y, \tau), V \approx \sum_{k=1}^{N} V_{k} \Phi_{k}(X, Y, \tau), \theta \approx \sum_{k=1}^{N} \theta_{k} \Phi_{k}(X, Y, \tau) C \approx \sum_{k=1}^{N} C_{k} \Phi_{k}(X, Y, \tau)
$$

The Galerkin finite element technique yields the subsequent nonlinear residual equations for the Eqs. (2), (3), (4) and (5), respectively at nodes of the internal domain $\Omega$ :

$$
\begin{aligned}
& R_{i}^{(1)}=\sum_{k=1}^{N} U_{k} \int_{\Omega}\left[\frac{\partial \Phi_{k}}{\partial \tau}+\left(\sum_{k=1}^{N} U_{k} \Phi_{k}\right) \frac{\partial \Phi_{k}}{\partial X}+\left(\sum_{k=1}^{N} V_{k} \Phi_{k}\right) \frac{\partial \Phi_{k}}{\partial Y}+\right] \Phi_{i} d X d Y d \tau- \\
& \xi\left[\sum_{k=1}^{N} U_{k} \int_{\Omega} \frac{\partial \Phi_{i}}{\partial X} \frac{\partial \Phi_{k}}{\partial X} d X d Y+\sum_{k=1}^{N} V_{k} \int_{\Omega} \frac{\partial \Phi_{i}}{\partial Y} \frac{\partial \Phi_{k}}{\partial Y} d X d Y\right]-\operatorname{Pr} \sum_{k=1}^{N} U_{k} \int_{\Omega}\left[\frac{\partial \Phi_{i}}{\partial X} \frac{\partial \Phi_{k}}{\partial X}+\frac{\partial \Phi_{i}}{\partial Y} \frac{\partial \Phi_{k}}{\partial Y}\right] d X d Y \\
& R_{i}^{(2)}=\sum_{k=1}^{N} V_{k} \int\left[\frac{\partial \Phi_{k}}{\partial \tau}+\left(\sum_{k=1}^{N} U_{k} \Phi_{k}\right) \frac{\partial \Phi_{k}}{\partial X}+\left(\sum_{k=1}^{N} V_{k} \Phi_{k}\right) \frac{\partial \Phi_{k}}{\partial Y}\right] \Phi_{i} d X d Y d \tau- \\
& \xi\left[\sum_{k=1}^{N} U_{k} \int_{\Omega} \frac{\partial \Phi_{i}}{\partial X} \frac{\partial \Phi_{k}}{\partial X} d X d Y+\sum_{k=1}^{N} V_{k} \int_{\Omega} \frac{\partial \Phi_{i}}{\partial Y} \frac{\partial \Phi_{k}}{\partial Y} d X d Y\right]-\operatorname{Pr} \sum_{k=1}^{N} V_{k} \int_{\Omega}\left[\frac{\partial \Phi_{i}}{\partial X} \frac{\partial \Phi_{k}}{\partial X}+\frac{\partial \Phi_{i}}{\partial Y} \frac{\partial \Phi_{k}}{\partial Y}\right] d X d Y \\
& -\operatorname{Pr} \operatorname{Ra}\left[\int_{\Omega}\left(\sum_{k=1}^{N} \theta_{k} \Phi_{k}\right) \Phi_{i} d X d Y-N r \int\left(\sum_{\Omega=1}^{N} C_{k} \Phi_{k}\right) \Phi_{i} d X d Y\right]
\end{aligned}
$$




$$
\begin{aligned}
& R_{i}^{(3)}=\sum_{k=1}^{N} \theta_{k} \int_{\Omega}\left[\frac{\partial \Phi_{k}}{\partial \tau}+\left(\sum_{k=1}^{N} U_{k} \Phi_{k}\right) \frac{\partial \Phi_{k}}{\partial X}+\left(\sum_{k=1}^{N} V_{k} \Phi_{k}\right) \frac{\partial \Phi_{k}}{\partial Y}\right] \Phi_{i} d X d Y d \tau- \\
& \frac{1}{\operatorname{Pr} R e} \sum_{k=1}^{N} \theta_{k} \int_{\Omega}\left[\frac{\partial \Phi_{i}}{\partial X} \frac{\partial \Phi_{k}}{\partial X}+\frac{\partial \Phi_{i}}{\partial Y} \frac{\partial \Phi_{k}}{\partial Y}\right] d X d Y-D_{f} \sum_{k=1}^{N} C_{k} \int_{\Omega}\left[\frac{\partial \Phi_{i}}{\partial X} \frac{\partial \Phi_{k}}{\partial X}+\frac{\partial \Phi_{i}}{\partial Y} \frac{\partial \Phi_{k}}{\partial Y}\right] d X d Y \\
& R_{i}^{(4)}=\sum_{k=1}^{N} C_{k} \int_{\Omega}\left[\frac{\partial \Phi_{k}}{\partial \tau}+\left(\sum_{k=1}^{N} U_{k} \Phi_{k}\right) \frac{\partial \Phi_{k}}{\partial X}+\left(\sum_{k=1}^{N} V_{k} \Phi_{k}\right) \frac{\partial \Phi_{k}}{\partial Y}\right] \Phi_{i} d X d Y d \tau- \\
& \frac{1}{S c R e} \sum_{k=1}^{N} C_{k} \int_{\Omega}\left[\frac{\partial \Phi_{i}}{\partial X} \frac{\partial \Phi_{k}}{\partial X}+\frac{\partial \Phi_{i}}{\partial Y} \frac{\partial \Phi_{k}}{\partial Y}\right] d X d Y-S_{r} \sum_{k=1}^{N} \theta_{k} \int_{\Omega}\left[\frac{\partial \Phi_{i}}{\partial X} \frac{\partial \Phi_{k}}{\partial X}+\frac{\partial \Phi_{i}}{\partial Y} \frac{\partial \Phi_{k}}{\partial Y}\right] d X d Y
\end{aligned}
$$

Three points Gaussian quadrature is used to evaluate the integrals in these equations. The non-linear residual Eqs. (8), (9), (10) and (11) are solved using Newton-Raphson method to determine the coefficients of the expansions in Eq. (7). The convergence of solutions is assumed when the relative error for each variable between consecutive iterations is recorded below the convergence criterion $\varepsilon$ such that $\left|\Psi^{n+1}-\Psi^{n}\right| \leq 10^{-4}$, where $n$ is the number of iteration and $\Psi$ is a function of $U, V, \theta$ and $C$.

\section{Mesh Generation}

In the finite element method, the mesh generation is the technique to subdivide a domain into a set of sub-domains, called finite elements, control volume, etc. The discrete locations are defined by the numerical grid, at which the variables are to be calculated. It is basically a discrete representation of the geometric domain on which the problem is to be solved. The computational domains with irregular geometries by a collection of finite elements make the method a valuable practical tool for the solution of boundary value problems arising in various fields of engineering. Fig. 2 displays the finite element mesh of the present physical domain.

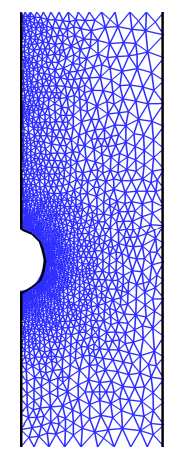

Fig. 2. Mesh generation of the sand.grain.

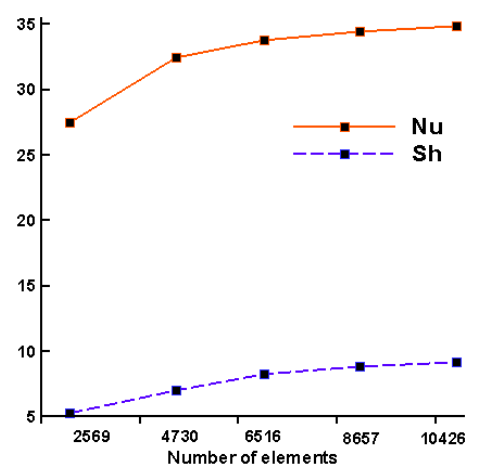

Fig. 3. Grid testing for the sand grain. 


\subsection{Grid testing}

An extensive mesh testing procedure is conducted to guarantee a grid-independent solution for $\operatorname{Ra}=10^{4}, \operatorname{Pr}=1.73, D_{f}=0.5$ and $S_{r}=0.5$ in a sand grain. In the present work, we examine five different non-uniform grid systems with the following number of elements within the resolution field: 2569, 4730, 6516, 8657 and 10426. The numerical scheme is carried out for highly precise key in the average Nusselt number $\mathrm{Nu}$ and Sherwood number $S h$ for the aforesaid elements to develop an understanding of the grid fineness as shown in Fig. 3. The scale of the average Nusselt number and Sherwood number for 8657 elements shows a little difference with the results obtained for the other elements. Hence, considering the non-uniform grid system of 8657 elements is preferred.

\subsection{Code validation}

The present numerical solution is validated by comparing the current code results for streamlines, isotherms and concentration at $R=0.5, N=1, D_{f}=S_{r}=0.5, S c=5, \operatorname{Pr}=$ 11.573 and $R a_{T}=10^{5}$ with the graphical representation of Nithyadevi and Yang [29] which was reported for Double diffusive natural convection in a partially heated enclosure with Soret and Dufour effects. Fig. 4 demonstrates the above stated comparison. The numerical solutions (present work and Nithyadevi and Yang [29]) are in good agreement.
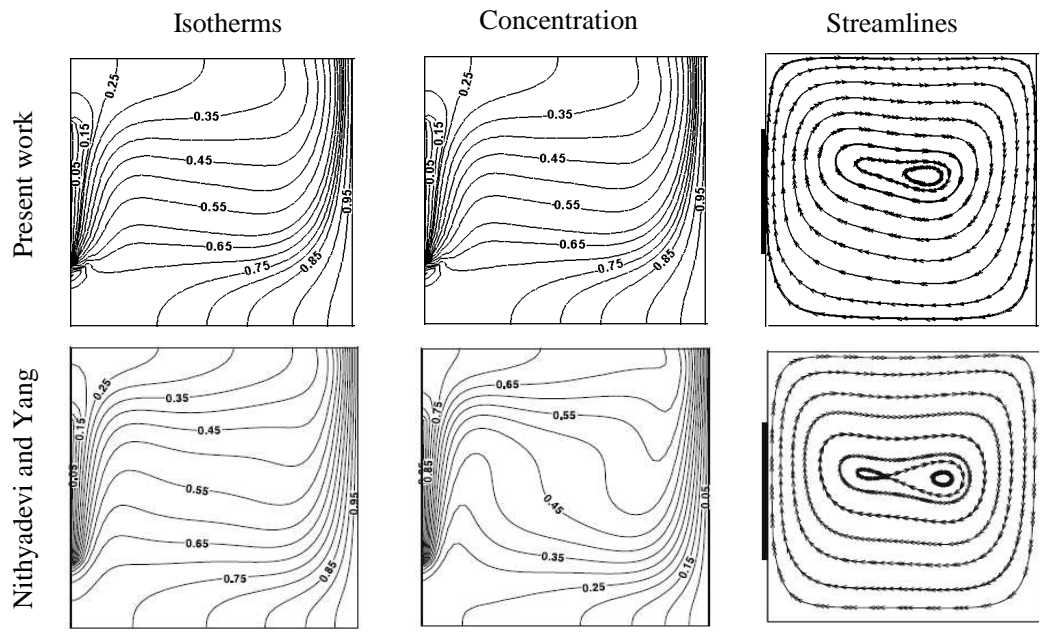

Fig. 4. Comparison between present work and Nithyadevi and Yang [29].

\section{Results and Discussion}

The model is an axially symmetric fluid-flow simulation in a moving coordinate system. In this section, numerical results in terms of streamlines and isotherms for transient 
analysis are displayed, while Rayleigh number $\left(R a_{T}=R a_{c}\right)$, Prandtl number $\operatorname{Pr}$, Dufour coefficient $D_{f}$ and Soret coefficient $S_{r}$ are fixed at $10^{4}, 1.73,0.5$ and 0.5 respectively. Also, falling velocity and forces on the sand grain, the values of the local and average Nusselt and Sherwood numbers, average temperature, temperature at grain centre, $U$-velocity, $V$ velocity in the sub domain with non dimensional time have been calculated.

\subsection{Velocity field}

The velocity field (modulus of the velocity vector) in terms of streamlines is displayed in Fig. 5. The occurring non-dimensional time of undershoot is chosen as the measure for the development time of the convective flow. The series of snapshots are of the velocity field, from a moment just after the sand grain is released until it is approaching steady state. Notice that the velocities are plotted in the reference system of the sand grain. Firstly, the plot shows that the streamlines are identical ie, there is no perturbation in velocity field. The deviation slightly grows as the time is increased. Due to time passing, the sand grain accelerates from standstill and the recirculation forms above the grain. Then created vortex enhances in size sequentially and rapidly reaches its terminal velocity. Finally after nondimensional time $\tau=0.1$, the velocity field becomes steady.

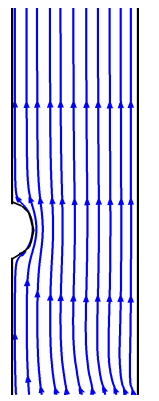

$\tau=0.001$

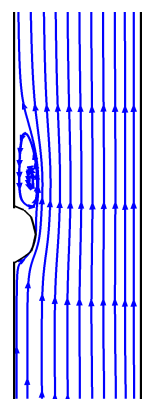

$\tau=0.06$

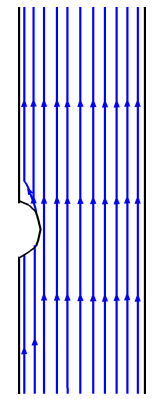

$\tau=0.01$

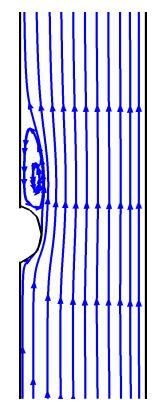

$\tau=0.07$

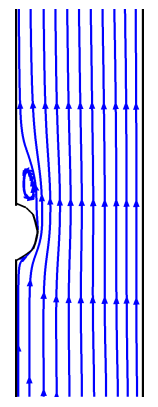

$\tau=0.02$

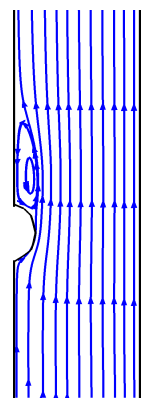

$\tau=0.08$

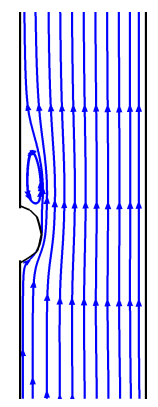

$\tau=0.03$

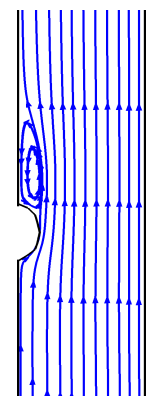

$\tau=0.09$
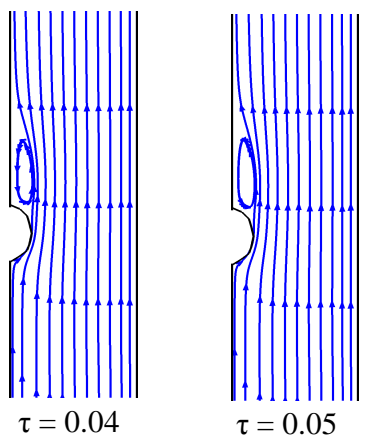

$\tau=0.05$

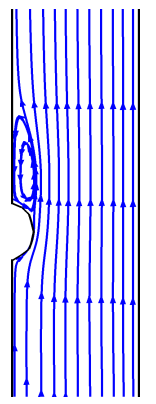

$\tau=0.1$

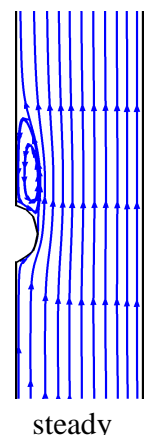

Fig. 5. Streamlines with different non-dimensional. 


\subsection{Temperature field}

Fig. 6 depicts the thermal development as a function of time for the sand grain. At the primary stage of time the isothermal lines occupy the bulk of the sand grain. The isotherms tend to move from right wall to the left insulated surface due to falling velocity of the grain. Gradually they are clustered near the lower hot surface as the time is increased. As well as the boundary layer thickness rises near the heated surface. At time $\tau>0.1$ there is no variation in the isothermal lines so that steady state pattern is observed.

\subsection{Concentration}

Fig. 7 shows the mass concentration phenomenon in terms of iso-concentration lines. It is observed from this figure that iso-concentrations are almost parallel to the active parts with compared to the isotherms indicating that both lines are spread out the whole sand grain and the convection is initiated. Increasing time, conduction mode of heat transfer is done. Further increasing time conduction is dominated across the grain for the terminal falling velocity of the solid particle. The iso-concentration lines tend to move from right highly concentrated wall to the left insulated surface. Then they are gathered near the lower concentrated bottom surface until the motion is steady.
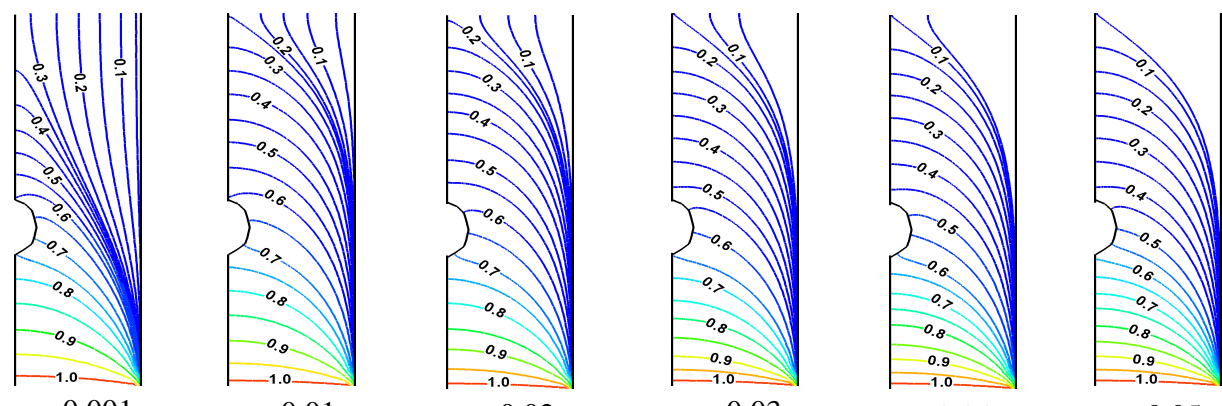

$\tau=0.001$

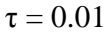

$\tau=0.02$

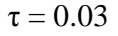

$\tau=0.04$
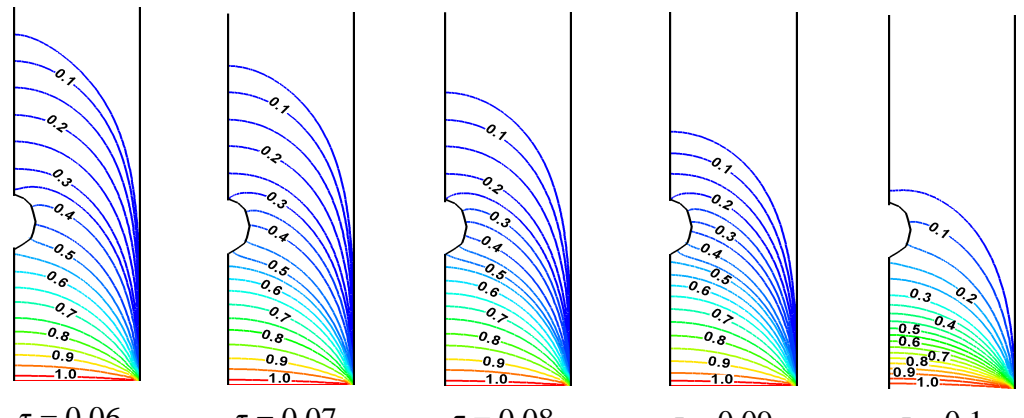

$\tau=0.05$

$\tau=0.06$

$\tau=0.07$

$\tau=0.09$

$\tau=0.1$

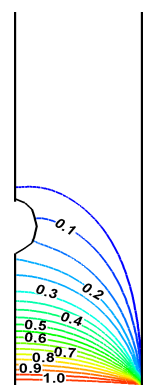

steady

Fig. 6. Isotherms with different nondimensional time. 


\subsection{Flow rate}

The falling velocity of the grain and the forces on the sand grain as a function of dimensionless time are exposed in Figs. 8 (i) - (ii). It is clear that after the solution time of 0.1 , the velocity approaches the terminal velocity. The terminal velocity equals $0.291 \mathrm{~m} / \mathrm{s}$. When this state is reached, the gravity and the forces from the water cancel out. From fig. 8(ii) we see that the fluid force ie the water exerts on the sphere (upper line) increases as the grain gains speed. The gravity force (lower line) remains the same, and the total force (middle line) tends toward zero as the solution approaches steady state.

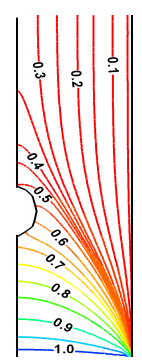

$\tau=0.001$

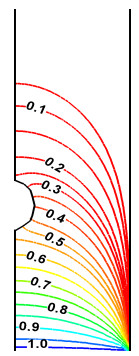

$\tau=0.06$

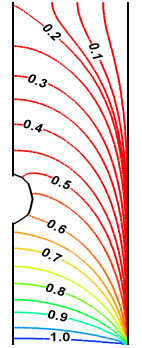

$\tau=0.01$

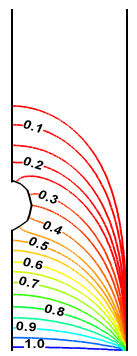

$\tau=0.07$

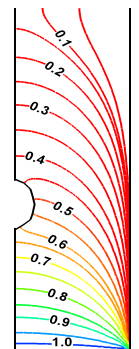

$\tau=0.02$

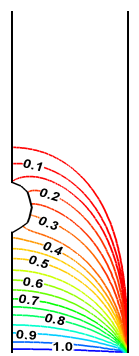

$\tau=0.08$

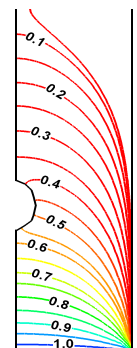

$\tau=0.03$

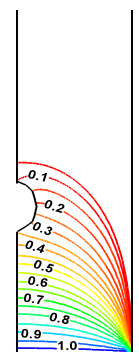

$\tau=0.09$

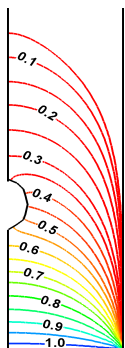

$\tau=0.04$

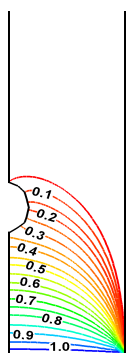

$\tau=0.1$

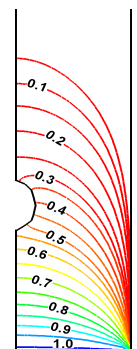

$\tau=0.05$

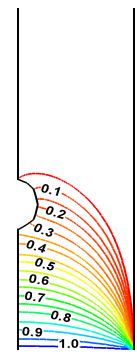

steady

Fig. 7. Iso-concentrations with different nondimensional time.

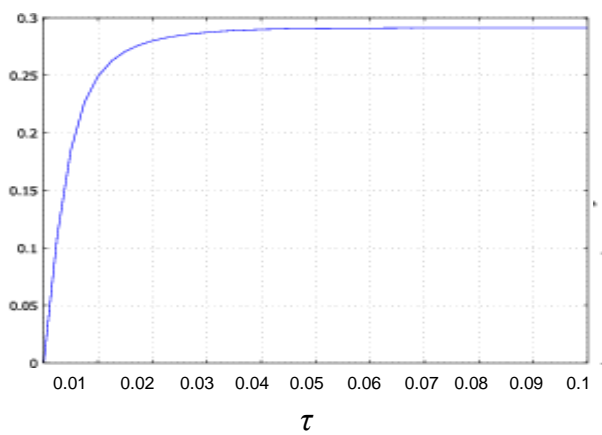

(i)

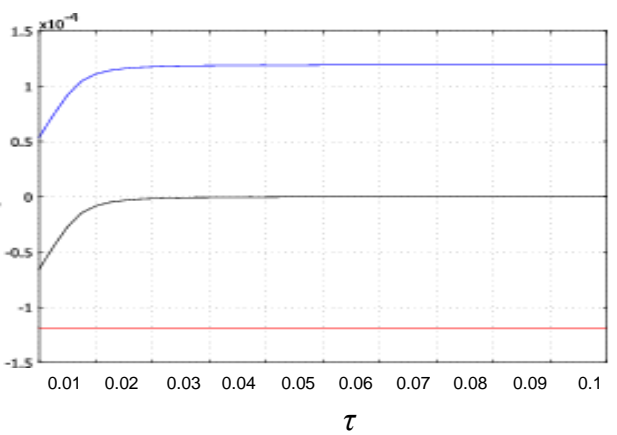

(ii)

Fig. 8. Plots of (i) falling velocity and (ii) the forces on the sand grain versus time. 


\subsection{Heat and mass transfer}

The local Nusselt number $\left(N u_{\text {local }}\right)$ and Sherwood number $\left(S h_{\text {local }}\right)$ along bottom hot and right vertical surfaces respectively and rate of heat $(\mathrm{Nu})$ and mass $(\mathrm{Sh})$ transfer versus time are presented in Figs. 9 (i) and (ii). With increasing $\tau$ up to 0.01 , the $N u_{\text {local }}$ and $S h_{\text {local }}$ increases and decreases respectively due to increment of temperature difference and decrement of thickness of boundary layer. Then both of them become invariant. The timeaveraged $\mathrm{Nu}$ and $\mathrm{Sh}$ are obtained by computing the time average of the average Nusselt and Sherwood number for a process, given by total time being the duration of the process of interest. $N u$ and $S h$ are the same pattern as $N u_{\text {local }}$ and $S h_{\text {local }}$ with time.

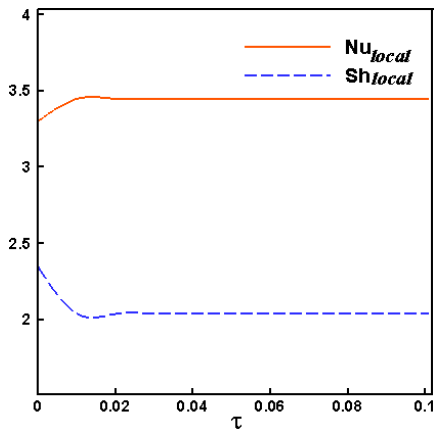

(i)

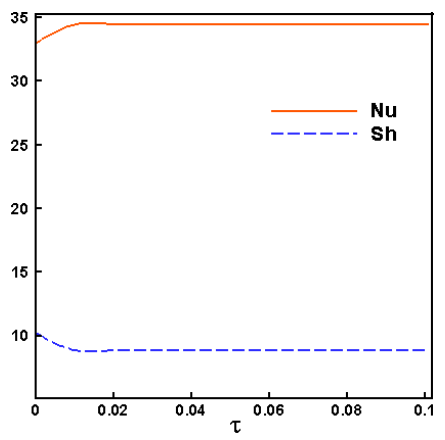

(ii)

Fig. 9. Plots of (i) local and (ii) average $N u$ and $S h$ at the respective surfaces versus time

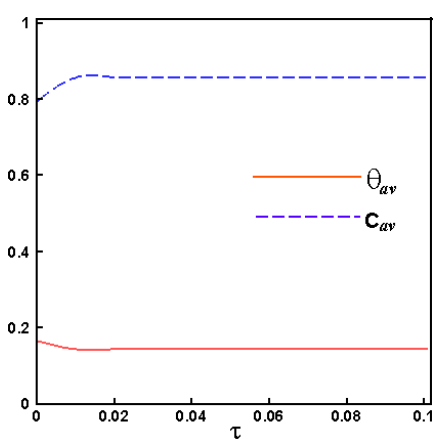

(i)

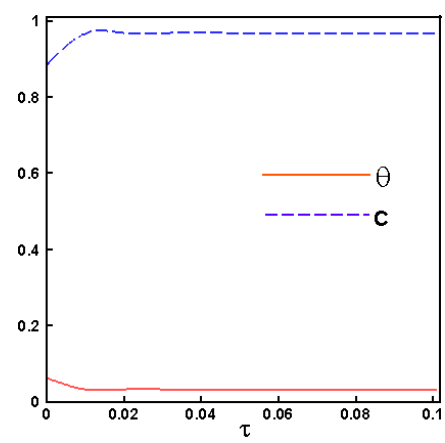

(ii)

Fig. 10. Plots of (i) $\theta_{a v}$ and $C_{a v}$ (ii) $\theta$ and $C$ at the centre of the subdomain versus time.

\subsection{Temperature and concentration}

Figs. 10 (i) and (ii) show the average fluid temperature and concentration versus time as well as temperature and concentration at subdomain centre of the sand grain. Average temperature $\left(\theta_{a v}\right)$ goes up but concentration $\left(C_{a v}\right)$ goes down for increasing values of time 
up to $\tau \leq 0.01$. Then they are almost identical with the variation of time. Temperature and concentration at the centre of the grain are also observed in similar pattern (Fig. 9).

\subsection{Velocity}

The $X$-velocity $(U)$ and Y-velocity $(V)$ in the subdomain of the sand grain as a function of time are illustrated in Figs. 11 (i)-(ii). It is seen that at $\tau=0.04$, the $U$ and $V$ velocities are of wavy pattern and increase sharply. Then these achieve optimum velocity gradually.

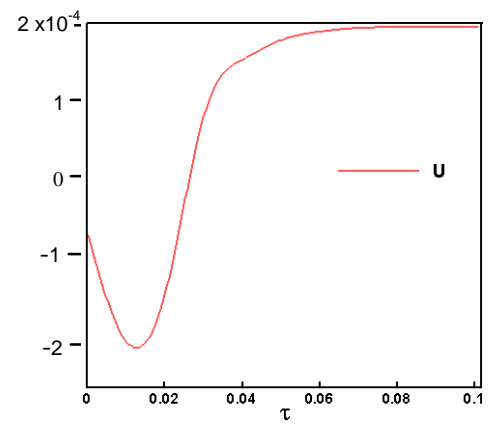

(i)

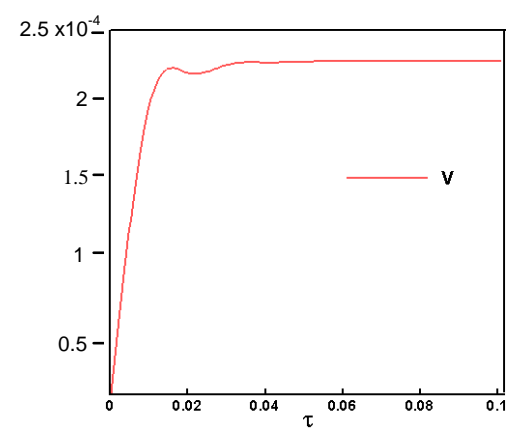

(ii)

Fig. 11. Plots of subdomain (i) X-velocity $U$ and (ii) Y-velocity $V$ versus dimensionless time.

\section{Conclusion}

The problem of transient falling behavior of a sand grain in the presence of Dufour and Soret effects has been studied numerically. Flow, temperature and concentration fields in terms of streamlines, isotherms and iso-concentration, respectively have been considered for various dimensionless times until the steady state observed. The results of the numerical analysis lead to the following conclusions:

a. The structure of the falling flow, temperature and concentration fields of the sand grain is found to be significantly dependent upon the time $(\tau)$.

b. The heat transfer rate is more effective than the rate of mass transfer.

c. The non-linear behavior of $U$ and $V$ velocities is due to the falling particle.

d. The mean temperature and concentration of the sand grain increase and decrease with dimensionless time, respectively.

One can easily adapt the model to hold for a general axially symmetric object (by redrawing the geometry) or even an arbitrarily shaped object (by modeling in 3D).

\section{References}

1. D. V. Davis, Int. J. Numer. Meth. Fluids 3, 249 (1983).

http://dx.doi.org/10.1002/fld.1650030305 
2. A. Ostrach, J. Heat Transfer 110, 1175 (1988). http://dx.doi.org/10.1115/1.3250619

3. W. J. Chang and D.F. Yang, Numer. Heat Transfer 28, 619 (1995). http://dx.doi.org/10.1080/10407789508913765

4. C. J. Ho, F. J. Tu, Int. J. Numer. Methods Heat Fluid Flow 11, 626 (2001). http://dx.doi.org/10.1108/EUM0000000005982

5. A. Osorio and R. Avila, J. Cervantes, Int. J. Heat Mass Transfer 47, 4491 (2004). http://dx.doi.org/10.1016/j.ijheatmasstransfer.2004.06.003

6. I. Sezai and A. A. Mohamad, Phys. Fluids 12, 2210 (2000). http://dx.doi.org/10.1063/1.1286422

7. S. Sivasankaran and P. Kandaswamy, Int. J. Fluid Mech. Res. 33, 345 (2006).

8. S. Sivasankaran and P. Kandaswamy, J. Korean Soc. Indus. Appl. Math. 11, 71 (2007).

9. F. Joly, P. Vasseur, and G. Labrosse, Int. Commun. Heat Mass Transfer 27, 755 (2000). http://dx.doi.org/10.1016/S0735-1933(00)00156-1

10. A. Bahloul, N. Boutana, and P. Vasseur, J. Fluid Mech. 491, 325 (2003). http://dx.doi.org/10.1017/S0022112003005524

11. A. Mansour, A. Amahmid, M. Hasnaoui, and M. Bourich, Num. Heat Transfer 49, 69 (2006).

12. M. K. Patha, P. V. S. N. Murthy, and G. P. Raja Sekhar, J. Heat Transfer 128, 605 (2006). http://dx.doi.org/10.1115/1.2188512

13. J. K. Platten, J. Appl. Mech. 73, 5 (2006). http://dx.doi.org/10.1115/1.1992517

14. R. L. Frederick and F. Quiroz, Int. J. Heat Mass Transfer 44, 1699 (2001). http://dx.doi.org/10.1016/S0017-9310(00)00219-2

15. B. Erbay, Z. Altac, and B. Sulus, Heat Mass Transfer 40, 909 (2004). http://dx.doi.org/10.1007/s00231-003-0497-x

16. H. F.Oztop, Int. J. Thermal Sci. 46, 149 (2007). http://dx.doi.org/10.1016/j.ijthermalsci.2006.04.009

17. N. Nithyadevi, P. Kandaswamy, S. Sivasankaran, Math. Prob.Eng. 2006, Article ID 23425, 16 pages (2006).

18. N. Nithyadevi and P. Kandaswamy, J. Lee, Int. J. Heat Mass Transfer 50, 4688 (2007). http://dx.doi.org/10.1016/j.ijheatmasstransfer.2007.03.050

19. N. Nithyadevi and R. J. Yang, Int. J. Heat Mass Transfer 52, 1667 (2009). http://dx.doi.org/10.1016/j.ijheatmasstransfer.2008.09.016

20. P. Kandaswamy, S. Sivasankaran, and N. Nithyadevi, Int. J. Heat Mass Transfer 50, 942 (2007). http://dx.doi.org/10.1016/j.ijheatmasstransfer.2006.08.013

21. A. J. Chamkha, Int. J. Energy 1 (3), 97 (2007).

22. M. A. Teamah, Int. J. Thermal Sci. 47 (3), 237 (2008). http://dx.doi.org/10.1016/j.ijthermalsci.2007.02.003

23. R. Nasrin, J. Sci. Res. 3 (3), 501 (2011). http://dx.doi.org/10.3329/jsr.v3i3.7433

24. S. Parvin and R. Nasrin, J. Sci. Res. 4 (2), 337 (2012). http://dx.doi.org/10.3329/jsr.v4i2.8142

25. R. Nasrin, J. Sci. Res. 4 (1), 39 (2012). http://dx.doi.org/10.3329/jsr.v4i1.8014

26. C. Taylor and P. Hood, Computer and Fluids 1 (1), 73 (1973). http://dx.doi.org/10.1016/0045-7930(73)90027-3

27. P. Dechaumphai, Finite Element Method in Engineering, $2^{\text {nd }}$ ed. (Chulalongkorn Univ. Press, Bangkok, 1999).

28. T. Basak, S. Roy, and I. Pop, Int. J. Heat Mass Transfer 52, 2471 (2009). http://dx.doi.org/10.1016/j.ijheatmasstransfer.2009.01.020

29. N. Nithyadev and R. -J. Yang, Int. J. Heat Fluid Flow 30, 902 (2009). http://dx.doi.org/10.1016/j.ijheatfluidflow.2009.04.001 